\author{
D-A 型多色电致变色材料的电化学合成与表征 \\ 侯影飞 $a$ 徐刚强 ${ }^{a, b}$ 赵金生*,b 孔摸 ${ }^{a}$ 杨冲 ${ }^{a}$ \\ ( ${ }^{a}$ 中国石油大学(华东)重质油国家重点实验室 青岛 266580) \\ ( ${ }^{b}$ 聊城大学化学化工学院 聊城 252059)
}

\begin{abstract}
摘要 设计合成了两种新型单体, 电化学聚合制得了相应 D-A 型聚合物: 聚 $\{10,13-$ 二(3,4-乙烯二氧噻吩基)二苯并 $[a, c]$ 吩嗪 $\}(\mathrm{P} 1)$ 和聚 $\{10,13$-二(4-乙基噻吩-2-基)二苯并 $[a, c]$ 吩嗪 $\}(\mathrm{P} 2)$. 研究发现, 聚合物膜 P1 在中性态呈现绿色, 且随着电 压的增加呈现多种不同的颜色, 其在 $1600 \mathrm{~nm}$ 时最大光学对比度达 $74.8 \%$, 响应时间为 $0.6 \mathrm{~s}$, 着色效率(CE)为 285.8 $\mathrm{cm}^{2} /$ C. 聚合物膜 P2 在氧化态和还原态时分别为灰蓝色和军绿色, 也具有较高的光学对比度, 快速的转换响应以及较 高的着色效率. 另外, 这两种导电聚合物都具有较低的光学禁带 $\left(E_{\mathrm{g}}\right)(\mathrm{P} 1$ 为 $1.26 \mathrm{eV}, \mathrm{P} 2$ 为 $1.54 \mathrm{eV})$. 本研究提供了两种具 有优良性能的电致变色材料, 并对其性能参数进行了分析研究.
\end{abstract}

关键词 二苯并 $[a, c]$ 吩嗪; 导电聚合物; D-A 性能; 电化学聚合; 电致变色

\title{
Electrosynthesis and Characterization of the Donor-Acceptor Type Multicolored Electrochromic Materials
}

\author{
Hou, Yingfei ${ }^{a} \quad$ Xu, Gangqiang ${ }^{a, b} \quad$ Zhao, Jinsheng*, $^{*} \quad$ Kong, Ying $^{a} \quad$ Yang, Chong ${ }^{a}$ \\ ( ${ }^{a}$ State Key Laboratory of Heavy Oil Processing, China University of Petroleum (East China), QingDao 266580) \\ ( ${ }^{b}$ Department of Chemistry and Chemical Engineering, LiaoCheng University, LiaoCheng 252059)
}

\begin{abstract}
Two novel monomer were synthesized via Stille cross coupling reaction. Electropolymerization of the monomers gave birth to the donor-acceptor type polymers: poly\{10,13-bis(2,3-dihydrothieno[3,4- $b][1,4]$ dioxin-5-yl) dibenzo[a,c]phenazine $\}$ (P1) and poly\{10,13-bis(4-ethylthiophen-2-yl)dibenzo[a,c]phenazine $\}$ (P2). Cyclic voltammetry and UV-vis spectroscopy were used to study the electrochemical and optical properties of the polymer films. The surface morphologies of the polymer films were investigated by scanning electron microscopy (SEM). The slight structural modification of the two polymer films results in the variation of the optical and electronic properties. As shown in the studies, P1 film was green in the neutral state, and with the increment of the applied potentials, four different colors were observed (light green, greyish-green, grey and cadet blue). The P1 film had prominent high optical contrast (T\%) of $74.8 \%$, excellent response time of $0.6 \mathrm{~s}$ and good coloration efficiency (CE) of $285.8 \mathrm{~cm}^{2} / \mathrm{C}$ at $1600 \mathrm{~nm}$. Electrochromic switching response for polymer film P1 at $470 \mathrm{~nm}, 790 \mathrm{~nm}$ was also monitored. The slight loss in percent transmittance contrast value at the three given wavelengths indicated the stable ability of the polymer film of P1. The colors of polymer film P2 in the neutral and oxidized state were greyish-blue and cadet green, respectively. The optical contrast (T\%) of P2 film was found to be $64.5 \%$, the response time was $2.4 \mathrm{~s}$ and the coloration efficiency (CE) was $316.7 \mathrm{~cm}^{2} / \mathrm{C}$ at the wavelength of $1340 \mathrm{~nm}$. P2 film showed high stability at the wavelength of $1340 \mathrm{~nm}$, but at $754 \mathrm{~nm}$ the stability was not satisfactory. Moreover, both of the two polymers exhibited low optical bandgap $\left(E_{\mathrm{g}}\right)$ (the $E_{\mathrm{g}}$ of $\mathrm{P} 1$ was $1.26 \mathrm{eV}$ and the $E_{\mathrm{g}}$ of $\mathrm{P} 2$ was $1.54 \mathrm{eV}$ ). Both polymer films revealed obvious donor-acceptor behaviors, and they were not only n-dopable but also p-dopable.

Keywords dibenzo $[a, c]$ phenazine; conducting polymers; donor-acceptor behavior; electrochemical polymerization; electrochromism
\end{abstract}

\section{1 引言}

电致变色材料可以随着施加电压的变化而改变其 光学性能. 目前, 电致变色材料在电致变色显示器件、 智能窗、无眩反光镜等领域得到了发展与应用 ${ }^{[14]}$. 有 机导电高分子聚合物材料越来越成为电致变色领域的
研究重点. 与传统的无机导电材料相比, 有机导电高分 子材料具有较高的稳定性、快速的响应时间、多重电致 变色性能并且结构容易改变等优点 ${ }^{[5]}$. 具有电致变色性 质的有机导电高分子包括聚苯胺、聚吡咯及聚噻吩类化 合物等，其中以聚噻吩类化合物为主，原因是噻吩的 3,4-位易于进行化学修饰, 可通过对噻吩类单体的化学

*E-mail: j.s.zhao@163.com; Tel.: 0635-8539607

Received June 13, 2014; published November 7, 2014.

Supporting information for this article is available free of charge via the Internet at http://sioc-journal.cn.

Project supported by the National Natural Science Foundation of China (No. 51473074), the General and Special Program of the Postdoctoral Science Foundation of China (Nos. 2013M530397, 2014T70861).

项目受国家自然科学基金(No. 51473074)、中国博士后基金面上及特别资助项目(Nos. 2013M530397, 2014T70861)资助. 
修饰来实现对噻吩聚合物的禁带、颜色及可溶性等多种 性质的调控 ${ }^{[6]}$. 在噻吩的 3,4-位引入长链的烷氧基分子 可以得到新的噻吩分子, 通过氧化聚合得到可溶性聚合 物, 可在红色中性态和蓝色氧化态之间转变(禁带: 2.04 $\mathrm{eV})^{[7]} .90$ 年代初德国 Bayer AG 实验室首次合成 3,4-乙 撑二氧噻吩(EDOT), 通过电化学或化学氧化聚合得到 的聚合物 PEDOT, 聚合物具有良好的导电性、稳定性、 光学特性及电致变色性质, 与聚噻吩相比, 禁带降低 $(1.6 \mathrm{eV})$, 薄膜中性态时呈现深蓝色, 氧化态时为透明 的浅蓝色 ${ }^{[6]}$. 后来证实, 通过噻吩分子的简单修饰难以 得到禁带低于 $1.6 \mathrm{eV}$ 的电致变色共轭聚合物. 2006 年以 来, Reynolds 等 ${ }^{[6]}$ 通过供体-受体(D-A)模式成功合成了 系列低禁带的电致变色聚合物. 其基本思想是含有规则 交替的电子供体-受体基团的共轭聚合物, 会导致其价 带和导带变宽, 从而使禁带降低. 在红、绿和蓝三基色 中, 中性态绿色最难得到, 需要分别在蓝色吸收区和红 色吸收区各有一个吸收峰, 并且吸收峰在氧化过程中同 时消失, 此类聚合物的禁带一般低于 $1.2 \mathrm{eV} .2004$ 年 Wudl 和 Sonmez 等 ${ }^{[8]}$ 采用 D-A 模式成功合成出第一种中 性态绿色膜. 近年来, Reynolds, Toppare 等 ${ }^{[9]}$ 课题组合 成出若干种中性态绿色的 D-A 型电致变色聚合物, 但时 至今日, 绿色膜依然很稀少, 特别是兼有多色性质的电 致变色聚合物依然受到欢迎.

被广泛研究的 D-A 型导电高分子聚合物的供电子 部分通常有噻吩单元、烷基噻吩单元、乙烯二氧基噻吩 单元、吡咯单元等 ${ }^{[10,11]}$, 供电子单元与不同的缺电子单 元相互作用构成了众多的电致变色导电聚合物. 对于缺 电子部分, 含有氮原子的苯并芳香杂环化合物的应用是 合成与设计这类系统的有效方法之一. 为此, 我们通过 Stille 偶合反应得到了两种聚合物单体 10,13-二(3,4-乙 烯二氧噻吩基)二苯并 $[a, c]$ 吩嗪(M1)和 10,13-二(4-乙基 噻吩-2-基)二苯并 $[a, c]$ 吩嗪(M2)(图 1), 并电化学聚合得 到了相应的聚合物 P1 和 P2. 经研究表明, 聚合物 P1 和 P2 都具有明显的 D-A 型导电高分子化合物的特点, 并 且都表现出了较低的光学禁带. 另外, 聚合物 P1在中性 态呈现绿色, 在不同电压下呈现了多重的电致变色性 能, 其最高的光学对比度达到 $74.8 \%$ (1600 $\mathrm{nm}$ 处), 同时 有着较高的稳定性和快速的响应时间. 因此, 聚合物 P1 在电致变色领域有着广泛的应用前景. 聚合物 P2 在掺 杂一去掺杂过程中表现出了三种不同的颜色, 其光学对 比度、响应时间、着色效率、稳定性等性能也比较令人 满意, 可以作为电致变色应用材料的潜在选择.

\section{2 结果与讨论}

\section{1 单体的合成}

\section{$2.1 .13,6$-二溴-1,2-苯二胺的合成 ${ }^{[12]}$}

将 4,7-二溴苯并噻二唑加入到无水乙醇溶剂中, 在 $0{ }^{\circ} \mathrm{C}$ 条件下搅拌, 分批加入还原剂 $\mathrm{NaBH}_{4}$. 反应结束后,
通过硅胶柱状色谱分离法进行分离提纯, 洗脱溶剂为正 己烷：二氯甲烷 $(V: V, 3: 1)$, 最后得到雪白色固体 3,6-二溴-1,2-苯二胺, 产率 78.2\%. ${ }^{1} \mathrm{H}$ NMR $\left(\mathrm{CDCl}_{3}, 400\right.$ $\mathrm{MHz}) \delta: 6.84(\mathrm{~s}, 2 \mathrm{H}), 3.88(\mathrm{~s}, 4 \mathrm{H})$ (Figure S1, Supporting Information).

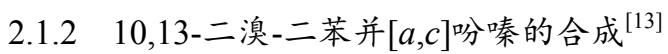

向一个装有回流管的圆底烧瓶中加入 3,6-二溴-1,2苯二胺、无水乙醇和菲醌. 3,6-二溴-1,2-苯二胺的加入量 与菲醌的相同. 催化剂量的对甲苯磺酸. 机械搅拌同时 加热回流反应混合物 $5 \mathrm{~h}$. 反应结束后, 减压旋蒸浓缩. 最后通过硅胶柱状色谱分离法进行分离提纯，洗脱溶剂 为正己烷: 二氯甲烷 $(V: V, 3: 1)$. 经分离提纯得到化 合物 10,13-二溴-二苯并 $[a, c]$ 吩嗪为黄色固体, 产率 85.7\%. ${ }^{1} \mathrm{H} \mathrm{NMR}\left(\mathrm{CDCl}_{3}, 400 \mathrm{MHz}\right) \delta: 9.46$ (d, $J=6.4 \mathrm{~Hz}$, $2 \mathrm{H}), 8.56(\mathrm{~d}, J=7.6 \mathrm{~Hz}, 2 \mathrm{H}), 8.03(\mathrm{~s}, 2 \mathrm{H}), 7.84(\mathrm{~m}, 4 \mathrm{H})$ (Figure S2, Supporting Information).

2.1 .3 单体 10,13 -二(3,4-乙烯二氧噻吩基 $)$ 二苯并 $[a, c]$ 吩嗪(M1)和 10,13-二(4-乙基噻吩-2-基)二苯并 $[a, c]$ 吩嗪 (M2)的合成 ${ }^{[13]}$

单体 M1 和 M2 通过 Still 偶合反应合成获得. 反应 需要加入催化剂 $\mathrm{Pd}\left(\mathrm{PPh}_{3}\right)_{2} \mathrm{Cl}_{2}$. 室温下通氮气搅拌 30 min. 之后, 加热到回流, 反应 $36 \mathrm{~h}$. 减压旋蒸浓缩. 最 后通过硅胶柱状色谱分离法进行分离提纯. 产品 M1 的 洗脱溶剂为正己烷: 二氯甲烷 $(V: V, 1: 1)$, 产品 $\mathrm{M} 2$ 的 洗脱溶剂为正己烷: 二氯甲烷 $(V: V, 2: 1)$. 纯化的产 品 $\mathrm{M} 1$ 为深红色固体, 产率 $84.9 \%$. m.p. $311{ }^{\circ} \mathrm{C} .{ }^{1} \mathrm{H} \mathrm{NMR}$ $\left(\mathrm{CDCl}_{3}, 400 \mathrm{MHz}\right) \delta: 9.62(\mathrm{~d}, J=9.2 \mathrm{~Hz}, 2 \mathrm{H}, \mathrm{ArH}), 8.73$ (s, 2H, ArH), 8.58 (s, 2H, ArH), $7.81 \sim 7.86(\mathrm{~m}, 4 \mathrm{H}, \mathrm{ArH})$, $6.71(\mathrm{~s}, 2 \mathrm{H}$, thienyl-H), 4.44 (dd, $J=4.4,4.8 \mathrm{~Hz}, 8 \mathrm{H}$, $\mathrm{OCH}_{2}$ ) (Figure S3, Supporting Information); HRMS (EI) calcd for $\mathrm{C}_{32} \mathrm{H}_{20} \mathrm{O}_{4} \mathrm{~S}_{2} \mathrm{~N}_{2}$ 560.6423, found 560.6399. Anal. calcd for $\mathrm{C}_{32} \mathrm{H}_{20} \mathrm{O}_{4} \mathrm{~S}_{2} \mathrm{~N}_{2}$ : C 68.6, S 11.4, N 5.0, O 11.4, H 3.6; found C 68.5, S 11.5, N 5.0, O 11.5, H 3.5. 纯化的产 品 $\mathrm{M} 2$ 为橘黄色固体, 产率 $85.2 \%$. m.p. $245{ }^{\circ} \mathrm{C} .{ }^{1} \mathrm{H} \mathrm{NMR}$ $\left(\mathrm{CDCl}_{3}, 400 \mathrm{MHz}\right) \delta: 9.57(\mathrm{~d}, J=6.4 \mathrm{~Hz}, 2 \mathrm{H}, \mathrm{ArH}), 8.58$ (d, $J=6.4 \mathrm{~Hz}, 2 \mathrm{H}, \mathrm{ArH}), 8.19$ (s, 2H, ArH), 7.78 (d, $J=$ $7.2 \mathrm{~Hz}, 6 \mathrm{H}, \mathrm{ArH}$ ), 7.26 (s, 2H, thienyl-H), 2.81 (q, $J=22.4$ $\mathrm{Hz}, 4 \mathrm{H}, \mathrm{CH}_{2}$ ), 1.39 (t, $J=14.8 \mathrm{~Hz}, 6 \mathrm{H}, \mathrm{CH}_{3}$ ) (Figure $\mathrm{S} 4$, Supporting Information); HRMS (EI) calcd for $\mathrm{C}_{32} \mathrm{H}_{24} \mathrm{~S}_{2} \mathrm{~N}_{2}$ 500.6764, found 500.6438; Anal. calcd for $\mathrm{C}_{32} \mathrm{H}_{24} \mathrm{~S}_{2} \mathrm{~N}_{2}$ : C 76.8, S 12.8, N 5.6, H 4.8; found C 76.7, S 12.8, N 5.7, H 4.8 .

\section{2 单体 M1 和 M2 的电化学性质及其聚合}

\subsection{1 单体 M1 和 M2 的电化学性质}

由图 2 所示, 这两种单体均呈现出双极性的氧化还 原性能. 在阳极扫描的过程中, 单体化合物 M1 的循环 伏安曲线在 $0.95 \mathrm{~V}$ 处展现了一个不可逆的氧化峰. 

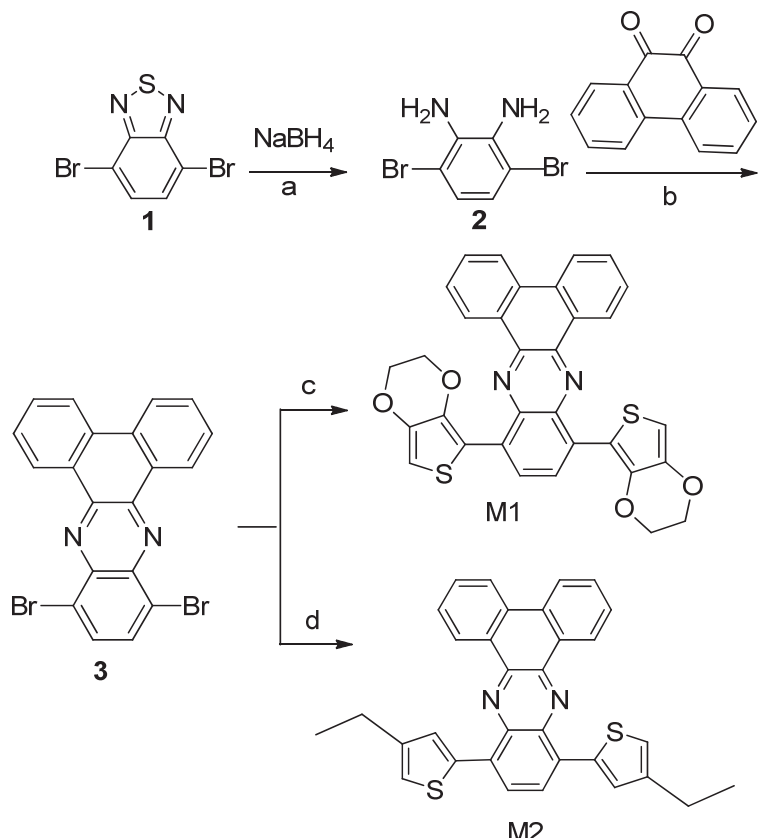

图 1 单体 $\mathrm{M} 1$ 和 $\mathrm{M} 2$ 的合成路线. (a) $\mathrm{NaBH}_{4}$, 乙醇, $0{ }^{\circ} \mathrm{C}$; (b) 对甲苯磺 酸, 回流; (c)或(d) $\mathrm{Pd}\left(\mathrm{PPh}_{3}\right)_{2} \mathrm{Cl}_{2}$, 甲苯, 回流

Figure 1 Synthetic routes of monomers M1 and M2. (a) $\mathrm{NaBH}_{4}$, ethanol, $0{ }^{\circ} \mathrm{C}$; (b) toluene- $p$-sulfonic acid, reflux; (c) or (d) $\mathrm{Pd}\left(\mathrm{PPh}_{3}\right)_{2} \mathrm{Cl}_{2}$, toluene, reflux

而单体化合物 M2 的不可逆氧化峰则出现在 $0.99 \mathrm{~V}$ 处. 在阴极扫描过程中, 两种单体均出现了明显的氧化还原 峰. 单体 M1 的还原峰半波电势比 M2 略低(M1 在- 1.34 $\mathrm{V}$ 处, M2 在 $-1.31 \mathrm{~V}$ 处). 由于单体 $\mathrm{M} 1$ 的分子共轭体系 与 M2 相比有更高的电子密度, 因此在形成 M1 阴离子 团时需要提供一个更负的电压来使电子进入共轭系 统 ${ }^{[14]}$. 这也恰好反映了单体 M1 的供电子部分(EDOT) 比 M2 的供电子部分(乙基噻吩)更富于电子.

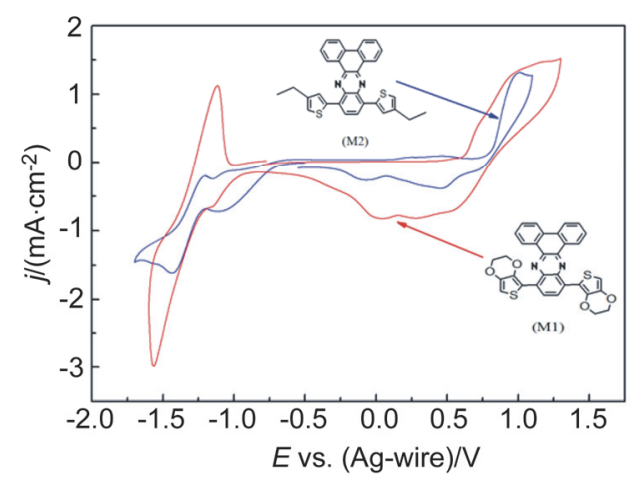

图 2 单体 $\mathrm{M} 1$ 和 $\mathrm{M} 2$ 在 $0.1 \mathrm{~mol} / \mathrm{L} \mathrm{TBAPF}_{6} / \mathrm{DCM}$ 溶液中的循环伏安图, 扫描速率为 $100 \mathrm{mV} \cdot \mathrm{s}^{-1}$

Figure 2 Cyclic voltammogram curves of monomers M1 and M2 in 0.1 $\mathrm{mol} / \mathrm{L} \mathrm{TBAPF} / \mathrm{DCM}$ at a scan rate of $100 \mathrm{mV} \cdot \mathrm{s}^{-1}$

\subsection{2 单体 $\mathrm{M} 1$ 和 $\mathrm{M} 2$ 的电化学聚合结果}

如图 3 所示, 聚合物 P1 的合成是通过在 -0.8 到 1.3 $\mathrm{V}$ 之间施加连续的电压扫描来完成的. 而聚合物 P2 的
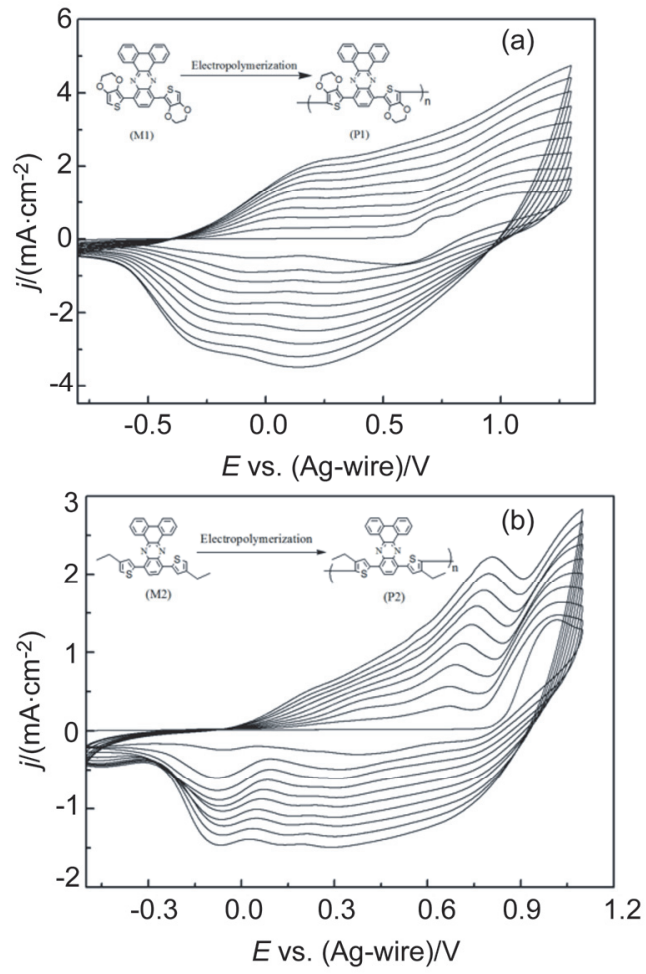

图 3 在 $0.1 \mathrm{~mol} / \mathrm{L} \mathrm{TBAPF} / / \mathrm{DCM}$ 溶液中 $0.003 \mathrm{~mol} / \mathrm{L} \mathrm{M} 1$ (a)和 0.003 $\mathrm{mol} / \mathrm{L} \mathrm{M} 2$ (b)单体的电化学聚合电势扫描曲线, 扫描速率为 $100 \mathrm{mV}$. $\mathrm{s}^{-1}$

Figure 3 Electropolymerization of $0.003 \mathrm{~mol} / \mathrm{L} \mathrm{M} 1$ (a) and $0.003 \mathrm{~mol} / \mathrm{L}$ $\mathrm{M} 2$ (b) in $0.1 \mathrm{~mol} / \mathrm{L} \mathrm{TBAPF}_{6} / \mathrm{DCM}$ solution at a scan rate of $100 \mathrm{mV} \cdot \mathrm{s}^{-1}$

扫描电压范围是在一 0.5 到 $1.1 \mathrm{~V}$ 之间. 随着氧化还原过 程的进行, 两者的电流密度呈现均匀有规律的增加, 同 时我们可以清晰的观察到工作电极上有聚合物的生成. 而氧化还原峰电流密度的增加表明沉积在工作电极上 的聚合物膜的量在增加 ${ }^{[15,16]}$. 另外, 单体 M1 的起始氧 化电压 $(0.61 \mathrm{~V})$ 比单体 M2 的起始氧化电压 $(0.84 \mathrm{~V})$ 低, 表明单体 M1 更容易被氧化.

\section{3 聚合物膜的表征}

\subsection{1 聚合物膜 P1 和 P2 的电化学表征}

用于研究聚合物氧化还原性能的聚合物膜是采用 循环伏安法循环三圈沉积在工作电极上制备得到的. 如 图 $4 \mathrm{a}$ 和图 $4 \mathrm{~b}$ 所示, 聚合物 P1 和 P2 均表现出了典型的 D-A 型导电聚合物的性质: 不仅具有稳定的 $p$-型掺杂过 程, 还表现出了明显的 $n$-型掺杂. $\mathrm{p}$-型掺杂过程中, 聚合 物 P1 在 $0.21 \mathrm{~V}$ 处有明显的氧化峰, 由其氧化还原过程 的不可逆性的原因，其对应的还原峰并不明显，在 0.38 $\mathrm{V}$ 左右有一个较宽的还原峰, 由于同样的原因, 其对应 的氧化峰也不明显. 聚合物 $\mathrm{P} 2$ 的氧化峰则处在 $0.80 \mathrm{~V}$, 还原峰在 $0.68 \mathrm{~V}$ 处. 聚合物膜的 $n$-型掺杂过程主要发生 在聚合物分子链的吸电子部分. 在 $n$-型掺杂过程中, P1 的氧化还原半波电势大约为 $-1.24 \mathrm{~V}, \mathrm{P} 2$ 的半波电势在 $-1.30 \mathrm{~V}$ 左右. 同时能够进行 $\mathrm{p}$-型以及 $\mathrm{n}$-型掺杂, 使聚 
合物 P1 和 P2 在电致变色器件、n-p 型晶体管等方面有 着更广泛的用途 ${ }^{[17]}$. 另外, 图 S5a 和图 S5b (Supporting Information) 分别为在 $\mathrm{p}$-型掺杂状态下的聚合物 P1与 P2 的峰电流密度与扫描速率之间关系. 峰电流密度与不同 扫描速率之间良好的线性关系表明电化学反应中的电 荷传递过程不受对阴离子扩散的控制, 同时也说明聚合 物膜良好的氧化还原活性以及与电极之间的良好的附 着性 ${ }^{[18]}$.
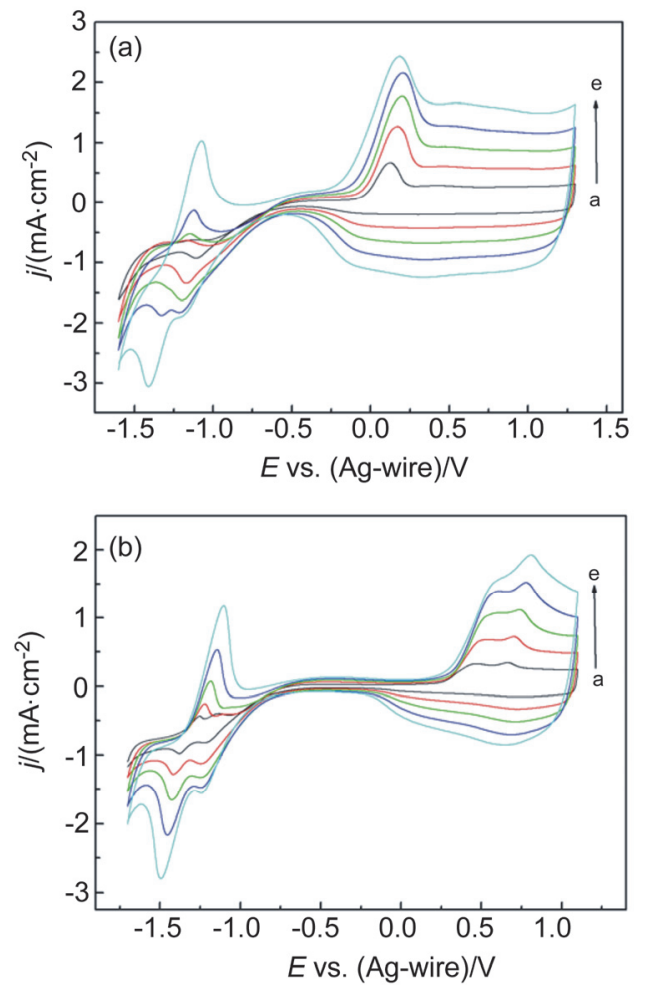

图 4 不同扫描速率下聚合物 P1 (a)以及聚合物 P2 (b)的特征循环伏 安曲线

Figure $4 \mathrm{CV}$ curves of polymer films P1 (a) and P2 (b) at different scanning rates between 50 to $250 \mathrm{mV} \cdot \mathrm{s}^{-1}$

(a) $50 \mathrm{mV} \cdot \mathrm{s}^{-1}$, (b) $100 \mathrm{mV} \cdot \mathrm{s}^{-1}$, (c) $150 \mathrm{mV} \cdot \mathrm{s}^{-1}$, (d) $200 \mathrm{mV} \cdot \mathrm{s}^{-1}$, (e) $250 \mathrm{mV} \cdot$ $\mathrm{s}^{-1}$

\subsection{2 单体和聚合物的光学性能表征}

中性状态下的沉积在 ITO 导电玻璃电极上的聚合 物膜以及溶解在 $\mathrm{CH}_{2} \mathrm{Cl}_{2}$ 溶液中的相应单体的 UV-vis 光 谱图通过 Varian Cary 5000 光谱仪测量得到. 如图 5 所 示，单体 M1 的主要最大吸收峰位于 $253 \mathrm{~nm}$ 以及 338 $\mathrm{nm}$ 处, 而相应的聚合生成的聚合物 P1 的最大吸收峰波 长发生红移, 处于 $470 \mathrm{~nm}$ 以及 $790 \mathrm{~nm}$ 处. 聚合物 $\mathrm{P} 2$ 的 最大吸收峰波长 $(350,550 \mathrm{~nm})$ 与相应单体 $\mathrm{M} 2$ 主要最大 吸收峰波长 $(252,318 \mathrm{~nm})$ 相比也同样发生了明显的红 移. 聚合物最大吸收峰的红移表明聚合物分子链的共轭 长度比相应单体的更大, 同时也证明了单体聚合的发 生.

另外, 从图 5 中我们可以得到单体以及聚合物的起

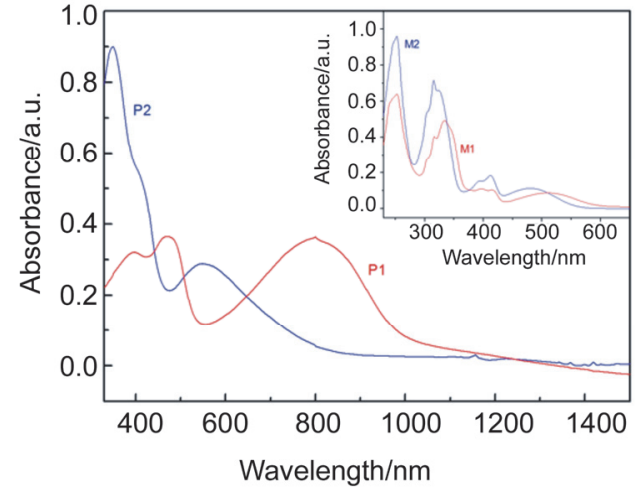

图 5 中性状态下沉积在 ITO 导电玻璃电极上的聚合物 P1 和 P2 的 UV-vis 光谱. 插图: 溶解在 $\mathrm{CH}_{2} \mathrm{Cl}_{2}$ 中的单体 $\mathrm{M} 1$ 和 $\mathrm{M} 2$ 的吸收光谱 Figure 5 UV-vis spectrum of polymer films P1 (a) and P2 (b) deposited on ITO electrode in the neutral state. Inset: absorption spectrum of monomers M1 and M2 dissolved in $\mathrm{CH}_{2} \mathrm{Cl}_{2}$

始吸收波长 $\left(\lambda_{\text {onset }}\right)$, 通过公式 $E_{\mathrm{g}}=1241 / \lambda_{\text {onset }}$ 还可以进一 步求得相应的光学禁带 $\left(E_{\mathrm{g}}\right)^{[19]}$. 单体 $\mathrm{M} 1$ 以及 $\mathrm{M} 2$ 的起始 吸收波长 $\left(\lambda_{\text {onset }}\right)$ 分别为 608 和 $572 \mathrm{~nm}$, 其相对应的光学 禁带 $\left(E_{\mathrm{g}}\right)$ 则分别为 2.04 和 $2.17 \mathrm{eV}$. 聚合物 $\mathrm{P} 1$ 和 $\mathrm{P} 2$ 的起 始吸收波长 $\left(\lambda_{\text {onset }}\right)$ 相对于单体也发生了红移(P1 为 987 $\mathrm{nm}, \mathrm{P} 2$ 为 $807 \mathrm{~nm})$, 因此其相对应的光学禁带 $\left(E_{\mathrm{g}}\right)$ 更低 $(\mathrm{P} 1$ 为 $1.26 \mathrm{eV}, \mathrm{P} 2$ 为 $1.54 \mathrm{eV})$. 比较低的光学禁带, 使电 子在聚合物共轭体系中最高已占有轨道(HOMO)与最低 未占有轨道的(LUMO)之间的跃迁更容易发生, 这对于 电致变色材料的应用有着重要的意义. 聚合物 P1 的光 学禁带相比于 P2 更低, 说明聚合物 P1 分子链的共轭程 度更好. 由于 EDOT 单元比乙基噻吩单元更富于电子, 因此 EDOT 的嵌入使分子链骨架中供电子-吸电子交替 共轭的共轭性更好. 另外, 乙基噻吩上的烷基链也会产 生一定的空间作用，使共轭链发生一定程度的扭曲，影 响共轭效果.

\subsection{3 聚合物膜的扫描电镜}

用于扫描电镜测试的聚合物膜是通过恒电位法沉 积在 ITO 导电玻璃上得到的, 并且在不含单体的空白溶 液中进行还原去掺杂. 如图 6 所示, 聚合物 P1 和 P2 的 膜表面均呈现出多孔结构. 聚合物 P1 膜呈现出海绵状 的形态, 而聚合物 P2 膜表面则表现的像成簇珊瑚状. 聚 合物表面的多孔结构有利于掺杂离子在掺杂-去掺杂过 程中自由的迁移进出聚合物膜, 而这能够提高聚合物膜 的响应时间，对聚合物膜的电致变色应用有重要意义.

\section{4 聚合物膜的电致变色性能}

\subsection{1 聚合物膜的光谱电化学性质}

聚合物膜的光电性能通过不同电压下的电子吸收 波谱的变化来探究. 在中性状态下, 聚合物 P1 膜在 470 $\mathrm{nm}$ (蓝光区)和 $790 \mathrm{~nm}$ (红光区)波长处有两个吸收峰，两 个吸收峰完全分开, 并且在 $554 \mathrm{~nm}$ 处呈现出谷底, 该 

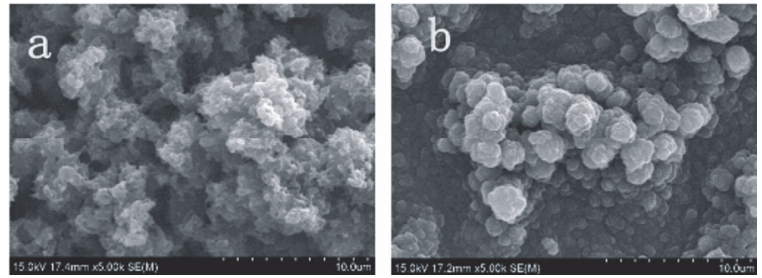

图 6 聚合沉积在 ITO 导电玻璃电极上的聚合物 P1 (a) 和 P2 (b)的扫 描电镜(SEM)图像

Figure 6 SEM images of P1 (a) and P2 (b) deposited onto ITO electrode

表 1 聚合物膜在中性, 氧化态和还原态下的 $L, a, b$ 值

Table 1 The values of $L, a, b$ of polymers in neutral, oxidized and reduced states

\begin{tabular}{cccccc}
\hline 聚合物 & $E$, vs. (Ag wire) $/ \mathrm{V}$ & $L$ & $a$ & $b$ & 颜色 \\
\hline \multirow{4}{*}{ P1 } & -0.8 & 54 & -11 & 35 & 绿色 \\
& 0.0 & 56 & -6 & 19 & 暗海蓝色 \\
& 0.2 & 57 & -5 & 12 & 暗海绿色 \\
& 0.5 & 58 & -2 & 11 & 灰色 \\
& 1.2 & 60 & -4 & 7 & 灰蓝色 \\
& -0.5 & 54 & -1 & 0 & 灰蓝色 \\
P2 & 0.5 & 49 & -4 & 6 & 灰绿色 \\
& 1.1 & 48 & -8 & 11 & 军绿色 \\
\hline
\end{tabular}

处峰谷窗口允许绿光透过(图 7a). 正是由于在可见光区 域的这两个吸收峰的存在, 聚合膜 P1 在中性状态下呈 现绿色. 聚合物 P1 在中性状态下的这两个吸收峰是由 于共轭体系的 $\pi-\pi^{*}$ 电子跃迁造成的. 但随着聚合物膜的 逐步氧化，这种跃迁强度逐渐减弱，最后初始的吸收峰 消失, 在 1030 和 $1600 \mathrm{~nm}$ 处出现了新的吸收峰(聚合物 膜的内部形成了极子和双极子). 因此, 随着测试电压 的增加，聚合物膜 P1 由绿色 $(-0.8 \mathrm{~V})$ 逐渐变为灰色 $(0.5$ $\mathrm{V})$, 最后变为灰蓝色 $(1.2 \mathrm{~V})$ (图 7a). 中性状态下聚合物 P2 的吸收峰在 350 和 $550 \mathrm{~nm}$ 处. 随着氧化的进行, 原 来的吸收峰消失, 在 754 和 $1340 \mathrm{~nm}$ 处出现了两个明显 的吸收峰. 还原态的聚合物 P2 为灰蓝色, 氧化态则变为 军绿色, 中间有较为明显的过渡色(图 7b).

中性态聚合物在低能量区的吸收峰取决于 D-A-D 型聚合物中电子供体向电子受体之间的电荷转移. 3,4乙撑二氧噻吩(EDOT) 是强供电子供体, 而吩嗪环是强 电子吸收体, 二者匹配良好, 聚合物 P1 在低能量吸收区 有较强的吸收峰, 并且和聚合物在高能量吸收区的强度 类似, 最终得到低禁带聚合物 ${ }^{[20]}$. 与 EDOT 相比, 3-乙 基噻吩的供电子能力较弱, 与吩嗪环的电荷转移作用较 弱, 这可从 P2 弱小的低能量吸收峰表现出来, 另外, 与 聚合物 P1 相比, 聚合物 P2 的起始吸收波长蓝移, 导致 聚合物 P2 的禁带升高. 聚苯胺在中性态时为黄色, 氧化 态(掺杂态)为蓝色, 而聚噻吩在中性态时为橙色(或红 色), 而氧化态时为蓝色, 且颜色变化单一. P1 和 P2 两
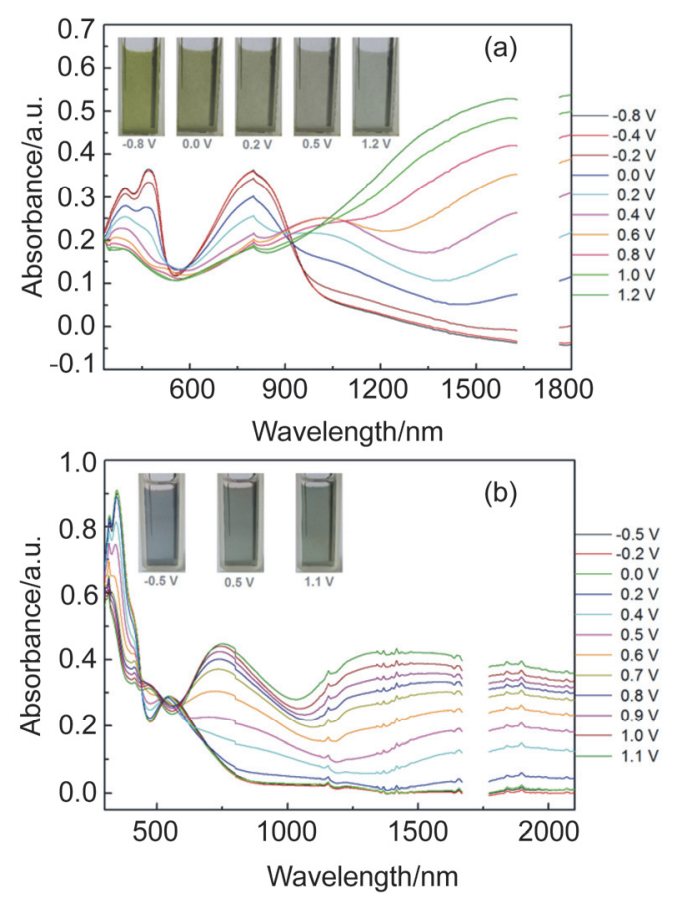

图 7 沉积在 ITO 导电玻璃上的聚合物膜 P1 (a) 和 P2 (b)在不同电压 下的光学吸收谱图

Figure 7 Spectroelectrochemical spectra of polymers P1 (a) and P2 (b) deposited on ITO electrode at different potentials

种聚合物膜均表现出了多重电致变色的性质, 特别是聚 合物膜 P1 在中性状态下为绿色，与中性态红色或中性 态蓝色膜相比, 中性态绿色膜难以得到(红绿蓝为加色 三基色，三基色相加可得到任何颜色). 为进一步辨别 聚合物的颜色，采用 CIE $1976 L \times a \times b$ 色度空间表示聚 合物在各个状态下的颜色, 结果如图 1 所示. 总之, 聚 合物 P1 及聚合物 P2 都具有低禁带、多色的特点, 在电 致变色显示器件的制备方面具有重要的应用前景.

\subsection{2 聚合物膜的电致变色动力学研究}

在掺杂态与完全的去掺杂态之间能够快速的转换 以及能够拥有较高的光学对比度, 对电致变色导电高分 子材料的实际应用有着重要的意义. 通过在 -0.5 与 1.1 $\mathrm{V}$ 之间施加阶跃时间为 $4 \mathrm{~s}$ 的阶跃电压, 研究沉积在导 电玻璃电极上的聚合物 $\mathrm{P} 1$ 的电致变色动力学性能. 聚 合物的光学对比度 $(T \%)$ 通常是指聚合物在某一单波长 下的掺杂-去掺杂过程中的透过率之差. 如图 8 所示, 聚 合物膜 P1 在 1600,790 以及 $470 \mathrm{~nm}$ 处的光学对比度 (T\%)分别为 $74.8 \%, 21.1 \%$ 和 $32.9 \%$, 特别是在 $1600 \mathrm{~nm}$ 波长处表现出了非常高的光学对比度. 另外, 在这三个 波长下聚合物 P1 经过 $300 \mathrm{~s}$ 的掺杂-去掺杂转换后其光 学对比度均没有明显的变化, 这表明聚合物 P1 膜具有 非常好的稳定性，并且能够很好的附着在导电玻璃电极 上. 聚合物 $\mathrm{P} 2$ 的阶跃电压被设定在 -0.8 到 $1.2 \mathrm{~V}$, 阶跃 时间同样为 $4 \mathrm{~s}$. 其在 $1340 \mathrm{~nm}$ 波长处的光学对比度 $(T \%)$ 为 $64.5 \%$, 且经过 $300 \mathrm{~s}$ 的电致变色转化后变化不 
明显, 但当测试波长为 $754 \mathrm{~nm}$ 时, 光学对比度 $(T \%)$ 仅 为 $12.2 \%$, 且随着时间的延长有明显的减小, 说明聚合 物 P2 膜稳定性没有 P1 好(图 S6, Supporting Information). 电致变色响应时间是指电致变色材料在掺杂-去掺杂过 程中达到 $95 \%$ 的完全变化时所用到的时间 ${ }^{[21]}$. 聚合物 P1 有着较快的电致变色响应, 其响应时间(由去掺杂状 态到掺杂状态)在 $1600 \mathrm{~nm}$ 时为 $0.6 \mathrm{~s}, 790 \mathrm{~nm}$ 时为 $0.5 \mathrm{~s}$, $470 \mathrm{~nm}$ 时为 $0.9 \mathrm{~s}$. 聚合物 P2 的响应时间 (由去掺杂状态 到掺杂状态)在 1340 和 $754 \mathrm{~nm}$ 分别为 $2.4 \mathrm{~s}$ 和 $1.1 \mathrm{~s}$.

另外, 我们还探究了这两种聚合物膜的电致变色着 色效率 $(\mathrm{CE})$. 着色效率 $(\mathrm{CE})$ 由光学密度的变化与单位面 积上的电荷量的比值得到. 可以按照公式 $\mathrm{CE}=$ $\Delta \mathrm{OD} / Q_{\mathrm{d}}$ 和 $\Delta \mathrm{OD}=\log \left(T_{\mathrm{b}} / T_{\mathrm{c}}\right)$ 计算求得 ${ }^{[21]} . \Delta \mathrm{OD}$ 表示聚 合物光学密度变化, $T_{\mathrm{b}}$ 和 $T_{\mathrm{c}}$ 分别为聚合物材料着色前后 的透过率, $Q_{\mathrm{d}}$ 代表单位面积上聚合物膜着色退色过程中 通过的电荷量. 聚合物 P1 膜在 1600, 790 和 $470 \mathrm{~nm}$ 处 的着色效率 $(\mathrm{CE})$ 分别为 $285.8,93.2$ 和 $114.6 \mathrm{~cm}^{2} / \mathrm{C}$. 聚合 物 $\mathrm{P} 2$ 膜在 $1340 \mathrm{~nm}$ 波长下的 $\mathrm{CE}$ 为 $316.7 \mathrm{~cm}^{2} / \mathrm{C}$, 而在 $754 \mathrm{~nm}$ 波长下则较小, 为 $58.3 \mathrm{~cm}^{2} / \mathrm{C}$.

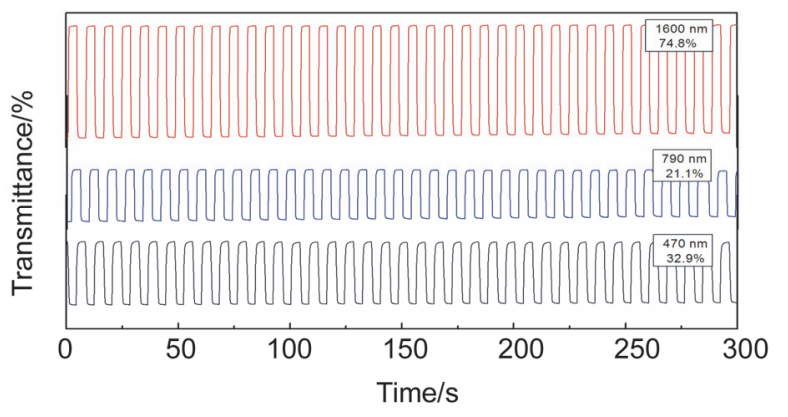

图 8 聚合物 P1 在 470, 790 和 $1600 \mathrm{~nm}$ 不同波长下, 阶跃时间为 $4 \mathrm{~s}$ 时的电致变色光学响应

Figure 8 Electrochromic switching response for polymer P1 monitored at 470,790 and $1600 \mathrm{~nm}$ with a residence time of $4 \mathrm{~s}$

\section{3 结论}

本实验成功合成得到了两种单体 $\mathrm{M} 1$ 和 $\mathrm{M} 2$, 并且 在 $0.1 \mathrm{~mol} / \mathrm{L} \mathrm{TBAPF}_{6} / \mathrm{DCM}$ 溶液进行电化学聚合, 得到 了相应的聚合物 P1 和 P2. 两种单体均呈现出了双极性 的氧化还原性能, 而相应的两种聚合物都既能进行 $n$ - 型 掺杂, 也能进行 $\mathrm{p}$-型掺杂, 表现出了明显 D-A 型导电聚 合物的特点. 实验中得到聚合物 P1 和 P2 的光学禁带 $\left(E_{\mathrm{g}}\right)$ 分别为 1.26 和 $1.54 \mathrm{eV}$, 由此可见两者均为低禁带化 合物. 多重的电致变色性能使这两种导电化合物在电致 变色领域更有应用的价值. 另外, 在稳定性方面, 聚合 物 P1 在各个波长处都表现出了较高的稳定性, P2 在 754 $\mathrm{nm}$ 处光学对比度随着扫描时间的增加有所降低. 在动 力学方面, 聚合物 P1 有着较高的光学对比度(其中在 $1600 \mathrm{~nm}$ 处最大为 $74.8 \%$ )、快速的响应时间以及较高的 着色效率. 聚合物 P2 在测试时也有着较好的表现. 总 之, 这两种聚合物(特别是聚合物 P1)在电致变色领域有
着重要的价值.

\section{4 实验部分}

\section{1 实验材料及仪器}

四氢呋喃(THF)使用前在 $\mathrm{Na}$ 二 二苯甲酮体系中干燥 回流除水. 有机锡化合物通过文献所述方法合成获 得 ${ }^{[9,10]}$. ITO 导电玻璃使用前需在超声波作用下用乙醇、 丙酮和去离子水依次进行清洗, 最后在氮气流中干燥. 实验中所用到的其他试剂都未经处理直接使用.

Varian AMX 400 光谱仪, APEXIII 7.0 TESLA FTMS 型质谱仪, Nicolet 5700 红外光谱仪, Varian Cary 5000 光谱仪, Thermo Finnigan Flash EA 1112 型元素分 析仪, Hitachi SU-70 热场发射扫描电子显微镜(SEM), Canon Power Shot A3000 IS 数码相机.

\section{2 实验方法}

单体电化学测试以及电化学聚合在一个连接电化 学工作站的一室三电极体系中进行. 工作电极、对电极、 参比电极分别是直径为 $0.5 \mathrm{~mm}$ 的铂丝、铂环和银丝. 假 参比电极用浓度为 $0.005 \mathrm{~mol} / \mathrm{L}$ 的二茂铁溶液 $\left(\mathrm{Fc} / \mathrm{Fc}^{+}\right)$进 行校准 ${ }^{[8]}$. 在含 $0.1 \mathrm{~mol} / \mathrm{L}$ 电解质六氟磷酸四丁基胺的二 氯甲烷溶液中, 银丝相对于饱和甘录的电势为 $0.03 \mathrm{~V}$. 聚合物光谱电化学的测试同样是在一个一室三电极装 置中进行的. 对电极和假参比电极仍然是铂环和银丝, 工作电极改变为 ITO 导电玻璃. 在有效面积为 $0.9 \mathrm{~cm} \times$ $1.9 \mathrm{~cm}$ 的 ITO 导电玻璃上进行电化学聚合, 得到可用于 电化学测试的聚合物膜. 所得的聚合物膜的厚度可以通 过控制流经体系的总电荷量来进行控制. 聚合物膜在进 行测试之前应先在不含单体的空白溶液中进行完全的 去掺杂. 光谱电化学数据可由一台与电脑相连的 Varian Cary 5000 型光谱仪测量得到.

\section{References}

[1] Xu, N.; Shen X. D.; Cui S. Rare Metals 2010, 34, 610.

[2] Li, M.; Yang, S.-W.; Zheng, J.-M.; Xu, C.-Y. Acta Chim. Sinica 2014, 72, 456. (李梅, 杨树威, 郑建明, 徐春叶, 化学学报, 2014, $72(4), 456$.

[3] Yang, S.-W.; Zheng, J.-M.; Wu, X.-M.; Xu, C.-Y. Acta Chim. Sinica 2013, 71, 1041. (杨树威, 郑建明, 吴星明, 徐春叶, 化学学报, 2013, 71, 1041.)

[4] Jiang, Y.; Wang, J.; Guan, L.; Zhong, Y.-P.; Liu, P.; Deng, W.-J. Acta Chim. Sinica 2012, 70, 103. (姜月, 王娟, 关丽, 钟一平, 刘 平, 邓文基, 化学学报, 2012, 70, 103.)

[5] Zhang, Z.-Y.; Yang, J.-P. Acta Chim. Sinica 2011, 69, 1247. (张增 阳, 杨继萍, 化学学报, 2011, 69, 1247.)

[6] Amb, C. M.; Dyer, A. L.; Reynolds, J. R. Chem. Mater. 2011, 23, 397.

[7] Xu, G. Q.; Zhao, J. S.; Cui, C. S.; Hou, Y. F.; Kong, Y. Electrochim. Acta 2013, 112, 95.

[8] Sonmez, G.; Shen, C. K. F.; Rubin, Y.; Wudl, F. Angew. Chem., Int. Ed. 2004, 43, 1498.

[9] Celebi, S.; Balan, A.; Epik, B.; Baran, D.; Toppare, L. Org. Electron. 2009, 10, 631 .

[10] Baran, D.; Oktem, G.; Celebi, S.; Toppare, L. Macromol. Chem. Phys. 2011, 212, 799.

[11] Özdemir, S.; Balan, A.; Baran, D.; Doğan, Ö.; Toppare, L. React. Funct. Polymer. 2011, 71, 168. 
[12] Tarkuc, S.; Udum, Y. A.; Toppare, L. Thin Solid Films 2012, 520, 2960.

[13] Bulut, U.; Kolay, M.; Tarkuc, S.; Toppare, L. J. Polym. Sci., Part A: Polym. Chem. 2011, 49, 3299

[14] Cihaner, A.; Alg1, F. Adv. Funct. Mater. 2008, 18, 3583.

[15] Xu, L. Y.; Zhao, J. S.; Cui, C. S.; Liu, R. M.; Liu, J. F.; Wang, H. S. Electrochim. Acta 2011, 56, 2815.

[16] Zhu, Z. J.; Xu, J. K.; Pei, M. S.; Lu, B. Y.; Ma, C.; Li, L.; Shen, L. Acta Chim. Sinica 2010, 68, 564. (朱召进, 徐景坤, 裴梅山, 卢宝
阳, 马茶, 李龙, 申亮, 化学学报, 2010, 68, 564.)

[17] Melek, P.; Tirkes, S.; Cihaner, A.; Alg1, F. Polymer 2010, 51, 62.

[18] Xu, C. X.; Zhao, J. S.; Wang, M.; Cui, C. S.; Liu, R. M. Thin Solid Films 2013, 527, 232

[19] Kaim, W.; Fiedler, J. Chem. Soc. Rev. 2009, 38, 3373.

[20] Akpınar, H.; Balan, A.; Baran, D.; Ünver, E. K.; Toppare, L. Polymer 2010, 51, 6123 .

[21] Hu, B.; Lv, X. J.; Sun, J. W.; Bian, G. F.; Ouyang, M.; Fu, Z. Y.; Wang, P. J.; Zhang, C. Org. Electron. 2013, 14, 1521. 